\section{Single nucleotide polymorphisms in PRSS1 and CASR genes are associated with chronic pancreatitis in North-Eastern region of India}

\section{To the Editor,}

The genetic risk factors of chronic pancreatitis (CP) include mutations in several genes, notably serine protease 1 (PRSS1), serine peptidase inhibitor (SPINK1), cystic fibrosis transmembrane conductance regulator (CFTR), cathepsin $B$ (CTSB), chymotrypsin $C$ (CTRC) and calcium sensing receptor (CASR). A genome wide association (GWAS) study in European populations revealed that polymorphisms in PRSS1 and claudin 2 (CLDN2) - MORC family CW-type zinc finger 4 (MORC4) locus were associated with CP [1]. Replication studies carried out globally, including two studies from north and south India have validated many findings of the GWAS $[2,3]$. No genetic data regarding CP were reported for the North-Eastern (NE) Indian population, as this population has a different genetic pattern than north or south India [4]. For the first time, we carried out a candidate gene association study in this population using previously validated markers significantly associated with CP in other populations.

Blood samples from $100 \mathrm{CP}$ patients as well as 110 nondiseased age matched controls without gastrointestinal diseases were collected from Indira Gandhi Memorial Hospital, Agartala. Total genomic DNA was isolated from blood cells, quality checked and targeted sequencing of 17 genes: PRSS1, PRSS2, SPINK1, CTRC, CTSB, CASR, CFTR, CLDN2, alcohol dehydrogenase $(A D H) 2$, aldehyde dehydrogenase 2 family member (ALDH) 1C, ALDH3A1, ALDH9A1,
ALDH4A1, cytochrome P450 family 2 subfamily E member 1 (CYP2E1), methylenetetrahydrofolate reductase MTHFR, glucuronosyltransferase family 1 member A7 (UGT1A7) at desired promoter, exons and UTR regions was done by the in-house Ion Torrent sequencing platform. Quality control steps excluded single nucleotide polymorphisms (SNPs) which were not in the Hardy Weinberg Equilibrium. Association analysis was done using PLINK, finally with $85 \mathrm{CP}$ cases and 78 controls to identify SNPs significantly associated with CP in the NE population by logistic regression after adjusting for confounders like age and sex. Allele-specific odds ratios at $95 \%$ CI were calculated. Meta-analysis was done using 'metafor' package in $\mathrm{R}$, using random effects model, publication bias being checked. Phasing of the genotypes was performed using SHAPEITv2, for which the "genetic map" was on build hg-19 from a $1000 \mathrm{G}$ database. Post phasing, the files were converted to PLINK input file format. Haplotype association was performed with PLINK-v1.07. The Haploreg and LDpair module of LDlink were used for the validation of linkage disequilibrium (LD) values and haplotype associations.

Association analysis of CP patients vs. controls showed three novel genetic variations in PRSS1 and CASR genes, the association being significant after adjustment for age and sex (Table I). As neither of these two SNPs in PRSS1 (rs6666 and rs6667) was reported before, we carried out haplotype association analysis. Interestingly, as shown in Fig. 1A, rs 10273639, the SNP in PRSS1 gene region, reported earlier by GWAS [1] and in both prior Indian studies $[2,3]$ was found in the same haplotype block together with rs6666 and rs6667. On the other hand, the CASR polymorphism (rs1801726) belongs to a set of polymorphisms clustered at the carboxy-terminal tail

Table I. Details of the SNPs in CASR and PRSS1, found to be associated with chronic pancreatitis

\begin{tabular}{|c|c|c|c|c|c|c|c|c|c|c|}
\hline \multirow[t]{2}{*}{ POSITION } & \multirow[t]{2}{*}{ SNP } & \multirow[t]{2}{*}{ GENE } & \multirow[t]{2}{*}{$\mathrm{A} 1$} & \multirow[t]{2}{*}{$\mathrm{A} 2$} & \multicolumn{2}{|c|}{ Frequency of $\mathrm{A} 1$ in } & \multirow[t]{2}{*}{ OR } & \multirow[t]{2}{*}{ SE } & \multirow[t]{2}{*}{ STAT } & \multirow[t]{2}{*}{$\mathrm{p}$} \\
\hline & & & & & Cases & Controls & & & & \\
\hline Chr3:122003832 & rs 1801726 & CASR & $\mathrm{C}$ & G & 0.92 & 0.96 & $0.27(0.08-0.90)$ & 0.61 & -2.14 & 0.03 \\
\hline Chr7:142460313 & rs6666 & PRSS1 & $\mathrm{T}$ & $\mathrm{C}$ & 0.75 & 0.6 & $0.56(0.32-0.97)$ & 0.28 & -2.06 & 0.04 \\
\hline Chr7:142460865 & rs6667 & PRSS1 & $\mathrm{T}$ & $\mathrm{C}$ & 0.75 & 0.6 & $0.56(0.32-0.97)$ & 0.28 & -2.06 & 0.04 \\
\hline
\end{tabular}


A

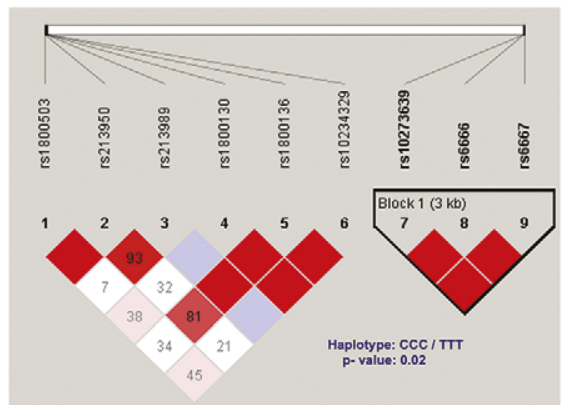

B

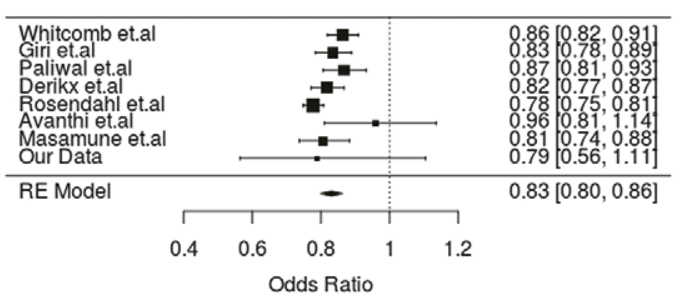

C

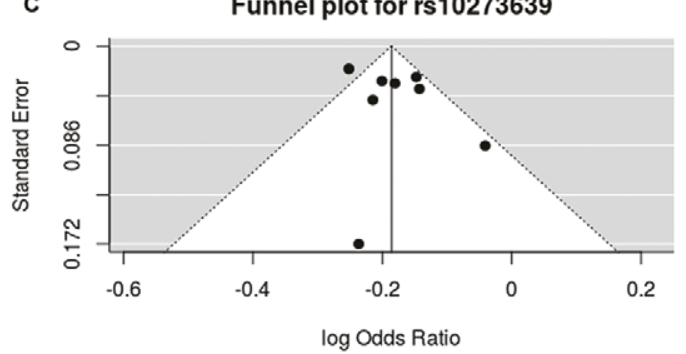

Fig. 1. A: Haplotype analysis with 9 SNPs in Chromosome 7 resulted in two haplotype blocks showing their Linkage Disequilibrium (LD) status. Among them, one haplotype block showing rs6666 and rs6667 is in LD with rs10273639 (as highlighted). B, C: Meta-analysis of rs10273639. B: Forest plot; C: Funnel plot.

where 'cytosine' to 'guanine' nucleotide change is responsible for the loss of function of CASR and hence elevation of the serum calcium level, feature of CP. Investigation for SNPs in LD with rs 1801726 resulted in the identification of a SNP in 3'-UTR of CASR (rs4677948). This SNP is associated with an elevated serum calcium level, as reported in ClinVar. We also found that the risk allele ' $\mathrm{A}$ ' at rs 4677948 created binding sites for three different miRNAs (miR-670-5p, miR-1236-3p and miR-877-3p) at 3'-UTR of CASR mRNA. miR-670 expression is upregulated in the pancreatic juice of pancreatic cancer patients and was also reported to promote cell proliferation in other cancers [5]. Interestingly, miR-877-3p also promoted apoptosis and it is known as an anti-autophagy miRNA [6]; both features could have a contributory role in the development of $\mathrm{CP}$. These results indirectly suggest that these miRNAs could have pro-inflammatory role and thereby justify their possible similar function in CP by targeting CASR mRNA. HaploReg analysis further showed that the SNP rs 1801726 creates a Pdx 1 binding site at the coding region of CASR, the implication of which is not clear right now.

Furthermore, when alcohol intake was also included as an independent factor in the logistic regression model and its effect adjusted, rs6666 and rs6667 were no longer significant, indicating that these SNPs are protective mainly for alcoholic CP. Though the GWAS study and other Indian studies found the PRSS1 polymorphisms to be retained in idiopathic CP patients, yet it was also observed by Whitcomb et al. (2012) [1], that PRSS1-PRSS2 locus (rs10273639) was associated mainly with alcoholic $\mathrm{CP}$, supporting our result.

In order to compare our association results with similar replication studies [1-3, 7-10], we carried out a meta-analysis of rs10273639. The ' $\mathrm{T}$ ' allele in rs10273639 was found to be protective whereas ' $C$ ' allele in rs 10273639 was found to enhance risk in all the studies used for meta-analysis. (Figs. 1B and $1 \mathrm{C}$ ). Our study followed the same trend with other reports despite small sample size. We did not find any significant association with reported SNPs in CLDN2 and SPINK1. Our results showed OR of $1.88(1.20-2.96)$ and $1.27(0.56-2.86)$ and allele frequency for risk allele ' $\mathrm{C}$ ' as 0.68 and 0.08 for rs7057398 in CLDN2 and rs17107315 in SPINK1, respectively, comparable to other studies. The lack of significant association could be due to small sample size.

In conclusion we identified novel single nucleotide polymorphisms in PRSS1 and CASR genes (rs6666, rs6667 and rs1801726) found to be associated with CP in a population of NE India. The SNP rs1801726 was in LD with another SNP in 3'-UTR of CASR, creating binding sites for pro-inflammatory miRNAs, miR-670-5p and miR-877-3p.

Bishnupriya Chhatriya ${ }^{1}$, Suman Kalyan Paine ${ }^{2}$, Sanchita Das ${ }^{3}$, Ankita Chatterjee ${ }^{1}$, Debashis Nath ${ }^{4}$, Ankur Mukherjee ${ }^{5}$, Analabha Basu $^{1}$, Srikanta Goswami ${ }^{1}$

1) National Institute of Biomedical Genomics, Kalyani, West Bengal;

2) ICMR- Regional Medical Research Centre, Dibrugarh, Assam;3) Institute of Post Graduate Medical Education and Research, Kolkata, West Bengal; 4) Department of Medicine, Indira Gandhi Memorial Hospital, Agartala, Tripura; 5) Cytel Statistical Software and Services pvt. Ltd., Pune, Maharashtra, India

Correspondence: Srikanta Goswami, srikanta_gs@hotmail.com sg1@nibmg.ac.in

Conflicts of interest: None.

Acknowledgement: We thank Prof. Partha Pratim Majumder, NIBMG for his guidance and to the Core Technology Research Initiative, NIBMG for technical support. The study has been supported by funding from the Department of Biotechnology, Government of India (Grant no. BT/231/NE/TBP/2011). BC received fellowship also from DBT-India

DOI: http://dx.doi.org/10.15403/jgld-788

\section{REFERENCES}

1. Whitcomb DC, LaRusch J, Krasinskas AM, et al. Common genetic variants in the CLDN2 and PRSS1-PRSS2 loci alter risk for alcohol-related and sporadic pancreatitis. Nat Genet 2012;44:1349-1354. doi:10.1038/ng.2466

2. Giri AK, Midha S, Banerjee P, et al. Common Variants in CLDN2 and MORC4 Genes Confer Disease Susceptibility in Patients with Chronic Pancreatitis. PloS One 2016;11:e0147345. doi:10.1371/journal. pone.0147345 
3. Paliwal S, Bhaskar S, Nageshwar Reddy D, et al. Association Analysis of PRSS1-PRSS2 and CLDN2-MORC4 Variants in Nonalcoholic Chronic Pancreatitis Using Tropical Calcific Pancreatitis as Model. Pancreas 2016;45:1153-1157. doi:10.1097/MPA.0000000000000608

4. Basu A, Sarkar-Roy N, Majumder PP. Genomic reconstruction of the history of extant populations of India reveals five distinct ancestral components and a complex structure. Proc Natl Acad Sci U S A 2016;113:1594-1599. doi:10.1073/pnas.1513197113

5. Wang J, Raimondo M, Guha S, et al. Circulating microRNAs in Pancreatic Juice as Candidate Biomarkers of Pancreatic Cancer. J Cancer 2014;5:696-705. doi:10.7150/jca.10094

6. Zhao X, Su L, He X, Zhao B, Miao J. Long noncoding RNA CA7-4 promotes autophagy and apoptosis via sponging MIR877-3P and MIR5680 in high glucose-induced vascular endothelial cells. Autophagy 2019;16:70-85. doi:10.1080/15548627.2019.1598750

7. Avanthi SU, Ravi Kanth VV, Agarwal J, et al. Association of claudin2 and PRSS1-PRSS2 polymorphisms with idiopathic recurrent acute and chronic pancreatitis: A case-control study from India. J Gastroenterol Hepatol 2015;30:1796-1801. doi:10.1111/jgh.13029

8. Derikx MH, Kovacs P, Scholz M, et al. Polymorphisms at PRSS1-PRSS2 and CLDN2-MORC4 loci associate with alcoholic and non-alcoholic chronic pancreatitis in a European replication study. Gut 2015;64:14261433. doi:10.1136/gutjnl-2014-307453

9. Masamune A, Nakano E, Hamada S, Kakuta Y, Kume K, Shimosegawa T. Common variants at PRSS1-PRSS2 and CLDN2-MORC4 loci associate with chronic pancreatitis in Japan. Gut 2015;64:1345-1346. doi:10.1136/ gutjnl-2015-309802

10. Weiss FU, Hesselbarth N, Parniczky A, et al. Common variants in the CLDN2-MORC4 and PRSS1-PRSS2 loci confer susceptibility to acute pancreatitis. Pancreatology 2018;5:477-481. doi:10.1016/j. pan.2018.05.486

\section{Fat droplets on direct cholangioscopy}

\section{To the Editor,}

Direct cholangioscopy (DC) with or without ancillary anchoring techniques is increasing in clinical use for high-quality visualization of biliary tract diseases and/or instrumentation under direct endoscopic vision. In this line, the work-up of indeterminate biliary strictures and cholangioscopy-guided treatment of complex stone disease remain first-line indications. By contrast, cholangioscopic assessment of freedom of stones has been reported to be superior to endoscopic retrograde cholangiography (ERC), in particular, in selected patients with diffuse bile duct dilation limiting fluoroscopy-based interpretation as the putative future gold standard. Contrast dye injection prior to cholangioscopy is notorious for interfering with visualization due to the formation of streak-like artifacts [1]. Here, we report on a novel phenomenon observed during DC reminiscent of biliary fat droplets, the distinct provenience and clinical relevance of which remain to be better determined.

Notwithstanding, albeit biliary fat secretion takes place on a "sub-endoscopic" level, it might be tempting to discuss an emulsion of contrast media and bile rich in cholesterol. In an elderly female patient with complex stone disease with status post cholecystectomy, several ERC sessions for papillotomy and presumed complete stone removal presented with increasing laboratory cholestasis and systemic inflammatory parameters, consistent with bacterial cholangitis. After the intake of antibiotics, the clinically stable patient underwent repeat ERC with DC in freehand fashion without $\mathrm{CO}_{2}$ and/or saline usage on day four. No purulent secretions were noted and an occlusion cholangiogram did not indicate remnant stones in the bile duct measuring $>25 \mathrm{~mm}$. This was confirmed on DC with full-scale visualization of the cystic duct remnant (Fig. $1 \mathrm{~A})$ as well as intrahepatic ducts up to a "wedged" position (Fig. 1B). In the common bile duct there were minor amounts of orange-colored bile fluid with intermingled fat droplets (Fig. 1C). Similar findings could be obtained in the right-hepatic ductal system with fat-containing globules flowing out of the deep biliary system (Fig.4D).

\section{Vincent Zimmer ${ }^{1,2}$, Frank Lammert ${ }^{2}$}

1) Department of Medicine, Marienhausklinik St. Josef Kohlhof, Neunkirchen; 2) Department of Medicine II, Saarland University Medical Center, Saarland University Homburg, Germany

Correspondence: Vincent Zimmer, vincent.zimmer@gmx.de
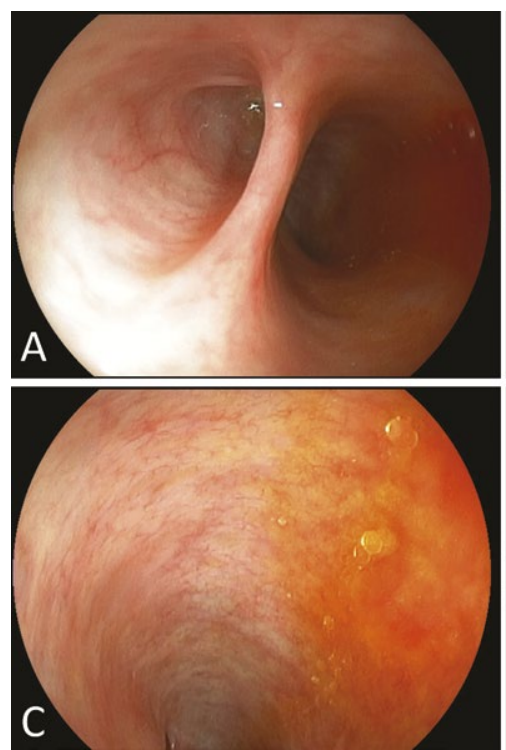

Fig. 1. (A) Direct cholangioscopic (DC) visualization of the cystic duct remanent (left) and the common hepatic duct (right) as well as (B) intrahepatic ducts (right anterior system) in a "wedged" position excluding remnant bile duct stone disease. (C) During advancement fat droplets were noted in the common bile duct (CBD) and (D) active flow of fat particles from deeper intrahepatic ducts was noted during intrahepatic cholangioscopy. 
Conflicts of interest: None.

DOI: http://dx.doi.org/10.15403/jgld-1116

\section{REFERENCES}

1. ASGE Technology Committee; Komanduri S, Thosani N, et al. Cholangiopancreatoscopy. Gastrointest Endosc 2016;84:209-221. doi:10.1016/j.gie.2016.03.013

\section{Sustained virological response following an 11-day course of direct acting antiviral therapy for hepatitis $\mathrm{C}$ infection}

\section{To the Editor,}

Direct acting anti-viral (DAA) medications have revolutionized the treatment of chronic hepatitis $\mathrm{C}(\mathrm{HCV})[1]$. Sustained virological response (SVR) can now be accomplished following 8-12 weeks of treatment in the majority of patients. Here we present a novel report of HCV eradication following only 11 days of treatment.

An asymptomatic 63-year-old non-cirrhotic female was referred for chronic HCV treatment at the liver clinic of Soroka University Medical Center. Medical history was significant for dyslipidemia, for which she stated that she was not taking medication. Laboratory workup showed elevated alanine aminotransferase (ALT) and aspartate aminotransferase (AST) of up to two times the normal upper limit. Virologic examination showed genotype $1 \mathrm{~b}$ and a viral load of 3,679,847 $\mathrm{IU} / \mathrm{ml}$. Serologic workup was significant to past exposure to hepatitis B virus (HBV) with development of protective immunity. The patient was prescribed treatment with Ombitasvir, Paritaprevir, Ritonavir and Dasabuvir for 12 weeks. On day 11 of treatment the patient began suffering from severe weakness and muscle rigidity and discontinued the treatment. On day 13 she presented to the emergency department after her muscle rigidity worsened. Blood tests revealed a creatinine kinase (CK) level of $8690 \mathrm{U} / \mathrm{L}$, AST of $759 \mathrm{U} / \mathrm{L}$, ALT of 457 $\mathrm{U} / \mathrm{L}$. The patient was admitted with a working diagnosis of rhabdomyolysis and supportive care with intravenous fluids was initiated. Upon further evaluation the patient recalled she had been concomitantly taking simvastatin for the past few weeks. Repeat testing did not disclose evidence of HBsAg reversion. After stopping simvastatin, CK, AST and ALT showed gradual decline. Following discharge, a HCV RNA test performed 30 days after treatment initiation was found to be negative and AST, ALT and CK were all within normal limits. Repeated HCV RNA testing at 12- and 24-weeks post treatment cessation were also negative. It was therefore concluded that the patient had obtained SVR.

This case report highlights HCV eradication following an ultra-short treatment course of only 11 days. Early discontinuation of treatment was mandated due to the wellknown drug-drug interaction between medications included in this regimen and simvastatin [2]. OATP/CYP3A4 inhibition by paritaprevir and ritonavir increases systemic simvastatin exposure which can subsequently lead to the development of myopathy and rhabdomyolysis. Simvastatin use was not disclosed by the patient at the time of DAAs initiation, which resulted in a potentially life-threatening outcome. The underlying mechanism for the rapid viral clearance seen in this case is not quite clear. One possible explanation is that the systemic inflammation induced by rhabdomyolysis triggered a hepatic inflammatory reaction which in turn enhanced HCV viral clearance. Alternatively, the abrupt elevation in liver enzymes could determined to the development of an idiosyncratic DAA-induced liver injury leading to accelerated viral clearance.

This report adds to accumulating data in the literature of HCV eradication following ultra-short DAAs therapy [3-5]. Further investigations are required to identify patients suitable for a shorter treatment course.

David Yardeni ${ }^{1}$, Vitaliy Dizengof ${ }^{1}$, Anat Nevo-Shor ${ }^{1}$, Naim Abu Freha $^{1}$, Harel Dahari ${ }^{2}$, Ohad Etzion ${ }^{1}$

1) Department of Gastroenterology and Liver Diseases, Soroka University Medical Center, Beer-Sheva, Israel; 2) The Program for Experimental and Theoretical Modeling, Division of Hepatology, Department of Medicine, Stritch School of Medicine, Loyola University Medical Center, Maywood IL, United States

Correspondence: David Yardeni, yardeda@gmail.com

Conflicts of interest: None.

DOI: http://dx.doi.org/10.15403/jgld-2153

\section{REFERENCES}

1. AASLD-IDSA. Recommendations for testing, managing, and treating hepatitis C. http://www.hcvguidelines.org. Accessed 9 Aug 2019.

2. Chauvin B, Drouot S, Barrail-Tran A, Taburet AM. Drug-drug interactions between HMG-CoA reductase inhibitors (statins) and antiviral protease inhibitors. Clin Pharmacokinet 2013;52:815-831. doi:10.1007/s40262-013-0075-4

3. Masetti M, Magalotti D, Martino E, Andreone P, Scuteri A, Zoli M. A Case of Acute Liver Failure during Ritonavir-Boosted Paritaprevir, Ombitasvir and Dasabuvir Therapy in a Patient with HCV Genotype 1b Cirrhosis. J Gastrointestin Liver Dis 2016;25:559-561. doi:10.15403/ JGLD.2014.1121.254.tti

4. Goyal A, Lurie Y, Meissner EG, et al. Modeling HCV cure after an ultra-short duration of therapy with direct acting agents. Antiviral Res 2017;144:281-285. doi:10.1016/j.antiviral.2017.06.019

5. Calvanese C, Lisotti A, Fiorenza S, Mandreoli M, Caletti G, Fusaroli P. Sustained virological response after 4-week ritonavir-boosted paritaprevir, ombitasvir and dasabuvir plus ribavirin treatment in a kidney transplant recipient. J Gastrointestin Liver Dis 2017;26:320-321. doi:10.15403/jgld.2014.1121.263.frz

\section{Homocysteine in nonalcoholic steatohepatitis: seemingly a paradox revisited}

\section{To the Editor,}

$\mathrm{Xu}$ et al. [1] reported that lower homocysteine levels were independently associated with nonalcoholic steatohepatitis (NASH) and significant fibrosis $(\mathrm{F} \geq 2)$, and were correlated 
with a higher grade of hepatocellular ballooning, albeit not with steatosis and lobular inflammation. Although being retrospective, this study is reportedly the largest to-date biopsyproven study on the association between homocysteine and nonalcoholic fatty liver disease (NAFLD) [1].

We also previously reported that lower homocysteine levels were independently associated with NASH, despite being similar between NAFLD patients and controls matched for gender, age, body mass index and waist circumference with patients [2] Likewise, homocysteine was lower by increasing the severity of fibrosis and portal inflammation, but not the steatosis grade, ballooning or lobular inflammation [2]. Subsequently, we incorporated homocysteine in a more integrated pilot analysis, leading to the introduction of a proposed term HSENSI (acronym of Homocysteine, Serum glutamic oxaloacetic transaminase [SGOT/AST], Erythrocyte sedimentation rate [ESR], Nonalcoholic Steatohepatitis Index), as a noninvasive marker differentiating NASH from simple steatosis (nonalcoholic fatty liver; NAFL) [3]; according to HSENSI, the lower the homocysteine and the higher the AST and ESR, the probability of NASH was higher [3]. In a post-hoc analysis of the same study, homocysteine was inversely associated with irisin levels in NAFLD patients, but not in the controls [4].

Our study [2] had a limited number of NAFLD patients compared with the $\mathrm{Xu}$ et al. study (31 vs. 289 , respectively) [1]. It was a small sample being, however, sufficient to provide statistically significant similar associations, at least between homocysteine and NASH or fibrosis. However, our study was also specifically designed for the association between homocysteine and NASH and included 22 matched controls [2], contrary to the uncontrolled, retrospective study, acknowledged by Xu et al. [1]. Due to probable misconception, $\mathrm{Xu}$ et al. reported 39 rather than 31 NAFLD patients in our study and that homocysteine was "unrelated to NAFLD biopsy", which is not the fact, as mentioned above $[2,4]$.

We have no mechanistic evidence of the lower homocysteine levels in NASH rather than symple steatosis (SS). However, because homocysteine levels provide substrate for glutathione repletion, we previously hypothesized that lower homocysteine in NASH may represent a counteracting effort of hepatocytes to increase glutathione through the transsulfuration pathway. Since NASH is a state of high oxidative stress, the intensification of synthesis of the antioxidant glutathione may mirror a self-defense mechanism of the hepatocytes, thus resulting in homocysteine depletion [2]. However, this remains to be demonstrated, since the observational design of our and $\mathrm{Xu}$ et al. studies does not allow cause-effect extractions $[1,2]$.

A meta-analysis of eight observational studies published in 2016, showed higher homocysteine levels in NAFLD patients than controls [5]. Nevertheless, there was high heterogeneity in this meta-analysis, which was not explained (e.g. by performing meta-regression). More importantly, a comparison between NAFL and NASH patients was not performed, possibly owing to the limited number of studies with histologically confirmed NAFLD. However, the main clinical question is the noninvasive diagnosis between NAFL and NASH, which currently requires liver histology. In this regard, the Xu et al. study adds to the literature and warrants mechanistic studies and specifically designed clinical studies with biopsy-proven NAFLD [1].
NAFLD is a multifactorial disease, thus diverse factors contribute to its development and progression on an individual basis. Insulin resistance is a prevailing contributing factor, so higher homocysteine levels might have been expected in NASH rather than NAFL, since homocysteine is associated with insulin resistance in other diseases. However, it seems that at least some NASH patients have lower than expected homocysteine levels, a seemingly paradox that is revisited following the Xu et al. [1] study and which gives birth to many scientific questions with possible clinical implication.

Stergios A. Polyzos ${ }^{1}$, Apostolis Papaefthymiou ${ }^{1}$, Michael Doulberis $^{1}$, Panagiotis Katsinelos ${ }^{2}$, Jannis Kountouras ${ }^{2}$

1) First Laboratory of Pharmacology, School of Medicine, Aristotle University of Thessaloniki, Thessaloniki; 2) Second Medical Clinic, School of Medicine, Aristotle University of Thessaloniki, Ippokration Hospital, Thessaloniki, Greece

Correspondence: Stergios A. Polyzos, spolyzos@auth.gr

Conflicts of interest: None.

DOI: http://dx.doi.org/10.15403/jgld-1667

\section{REFERENCES}

1. Xu Y, Guan Y, Yang X, Xia Z, Wu J. Association of Serum Homocysteine Levels with Histological Severity of NAFLD. J Gastrointestin Liver Dis 2020;29:51-58. doi:10.15403/jgld-529

2. Polyzos SA, Kountouras J, Patsiaoura K, et al. Serum homocysteine levels in patients with nonalcoholic fatty liver disease. Ann Hepatol 2012;11:68-76. doi:10.1016/S1665-2681(19)31488-7

3. Polyzos SA, Kountouras J, Slavakis A, et al. A novel noninvasive index for nonalcoholic steatohepatitis: a pilot study. Biomarkers 2013;18:607613. doi:10.3109/1354750X.2013.838305

4. Polyzos SA, Kountouras J, Anastasilakis AD, Margouta A, Mantzoros CS. Association between circulating irisin and homocysteine in patients with nonalcoholic fatty liver disease. Endocrine 2015;49:560-562. doi:10.1007/s12020-014-0473-X

5. Dai Y, Zhu J, Meng D, Yu C, Li Y. Association of homocysteine level with biopsy-proven non-alcoholic fatty liver disease: a meta-analysis. J Clin Biochem Nutr 2016;58:76-83. doi:10.3164/jcbn.15-54

\section{Inaction on IgA anti-tissue transglutaminase positivity}

\section{To the Editor,}

We read with considerable interest an article recently published in the Journal of Gastrointestinal and Liver Diseases by Henry et al. [1] on the importance of endoscopic biopsy in certain patient cohorts in their manuscript entitled "Seronegative Coeliac Disease Masquerading as Irritable Bowel Syndrome type Symptoms". They reported the case of a patient who was found to have coeliac disease on a duodenal biopsy despite serial negative serology. Henry et al. [1] concluded with the recommendation to perform duodenal biopsies in at risk patients despite normal serology owing to the 3-5\% rate of false negatives [2]. We think this is an astute and appropriate 
recommendation but would like to remind clinicians that duodenal biopsy is also important for the verification of a diagnosis of coeliac disease in the context of suggestive serology as per the British Society of Gastroenterology guidelines [3]. The positive predictive value of IgA anti-tissue transglutaminase (TTG) is in the region of only 54\% [4]. We have found a low rate of endoscopic verification of diagnosis of coeliac disease in a cohort of adult patients cared for within the catchment area of our institution in the South-East of Ireland. On auditing positive IgA anti-TTG results over an 18 month period from January 2018 to June 2019 we found that only $42 \%$ of patients had proceeded to endoscopic biopsy by April $1^{\text {st }} 2020$. This is a worrying outcome with possible adverse effects for the unverified patients with coeliac disease and the potentially presumptively diagnosed patients who have been committed to a needless lifelong insistence on avoidance of gluten containing foods. Reaching the correct diagnosis by the appropriate means is central to the practice of medicine. In the context of positive coeliac serology and that described by Henry et al., endoscopy is of fundamental importance in achieving a definitive diagnosis of coeliac disease. Our audit suggests this is being somewhat neglected by the clinician community at large within our catchment and should alert others to the potential for such practice within their surroundings.

\section{William Shanahan, Jayne Shanahan}

Department of Medicine, University Hospital Waterford, Co. Waterford, X91ER8E, Ireland

Corresponding to: William Shanahan, shanahaw@tcd.ie

Conflicts of interest: None.

Acknowledgements: University Hospital Waterford, Ireland. DOI: http://dx.doi.org/10.15403/jgld-2297

\section{REFERENCES}

1. Henry DX, Sanders DS, Basu K, Schiepatti A, Campbell JA. Seronegative Coeliac Disease Masquerading as Irritable Bowel Syndrome type Symptoms. J Gastrointestin Liver Dis 2020;29:111-113. doi:10.15403/jgld-718

2. Schiepatti A, Sanders DS, Biagi F. Seronegative coeliac disease: clearing the diagnostic dilemma. Curr Opin Gastroenterol 2018;34:154-158. doi:10.1097/MOG.0000000000000436

3. Ludvigsson JF, Bai JC, Biagi F, et al. Diagnosis and management of adult coeliac disease: Guidelines from the British society of gastroenterology. Gut 2014;63:1210-1228. doi:10.1136/gutjnl-2013-306578

4. Lock RJ, Stevens S, Pitcher MC, Unsworth DJ. Is immunoglobulin A anti-tissue transglutaminase antibody a reliable serological marker of coeliac disease? Eur J Gastroenterol Hepatol 2004;16:467-470. doi:10.1097/00042737-200405000-00005

\section{Reply,}

\section{To the Editor,}

We agree with the authors of the letter entitled "Inaction on IgA anti-tissue transglutaminase positivity" [1] on the importance of performing a duodenal biopsy for the diagnosis of adult coeliac disease (CD) and we thank them for offering us the possibility to address some methodological issues relevant for the diagnosis of CD.

Firstly, Shanahan et al. [1] reported that, over an 18-month period, only $42 \%$ of their patients who had previously tested positive to IgA tissue transglutaminase antibodies (anti-tTG) proceeded to a confirmatory duodenal biopsy. Despite this disappointing result, it would be interesting to have more details regarding the clinical phenotype of these patients such as presenting symptoms, levels of $\operatorname{IgA}$ anti-tTG, and results of endomysial antibodies (EmA), if performed as a confirmatory testing. Anyway, we have to acknowledge that failure to recognize $\mathrm{CD}$ at this step of the diagnostic process is still common. Endoscopy-related diagnostic errors in CD can be due either to the lack of performing a gastroscopy despite a highly suggestive clinical picture for $\mathrm{CD}$, or to inaccurate duodenal sampling during gastroscopy (which also includes not taking duodenal biopsies when CD is clinically suspected) [2-4]. Although ESPGHAN guidelines allow a biopsyavoidance diagnostic strategy in selected children [5], for the time being, in adults this approach is simply not applicable, and duodenal biopsy is still the mainstay for the diagnosis of adult CD [6].

We are not surprised by the unsatisfactory positive predictive value (PPV) of IgA anti-tTG (54\%) [7]. We previously reported a PPV for IgA anti-tTG of only $28.6 \%$ [2]. There may be several reasons for this. Firstly, positive anti-tTG have been found also in patients with liver disease, diabetes, end-stage heart failure and in non-coeliac children suffering from febrile infections [8-11]. Secondly, a reduced sensitivity for anti-tTG and EmA in patients with lesser degree of villous atrophy was reported $[2,12,13]$. Conversely, by using a higher cut-off value for anti-tTG, this leads to a higher PPV (75\%) in the same population under investigation [2]. Thirdly, assuming that sensitivity and specificity of a test are stable, the PPV is largely dependent on the pre-test probability for a certain disease in the population under examination. Therefore, we believe that in real life clinical practice the correct interpretation of a serological test for CD strictly depends on the expected prevalence (pre-test probability) of $\mathrm{CD}$ in a specific clinical population $[2,14,15]$. We previously showed that pre-endoscopy serological testing based on IgA anti-tTG in combination with duodenal biopsy of patients at high-risk of having $\mathrm{CD}$ (ie. patients with severe malabsorption or haematinic deficiencies) had a $100 \%$ sensitivity for the final diagnosis of CD. Similarly, this approach confidently identifies also those patients who likely do not require a duodenal biopsy, because they belong to a low-risk group and have a negative antibody testing for CD [14]. Interestingly, a recent prospective study has shown that measurement of deamidated gliadin peptide antibody before endoscopy can increase the detection of $\mathrm{CD}$ in an endoscopy setting with high pre-test probability for CD [15].

Finally, the diagnostic strategy of performing a gastroscopy with duodenal biopsy in patients with severe features of malabsorption, haematinic deficiencies and alarm symptoms despite negative coeliac antibodies is crucial in the diagnostic workup of seronegative CD and non-coeliac enteropathies 
[16-18]. In fact, despite being rare, these conditions are characterized by peculiar clinical phenotypes and a higher mortality than conventional seropositive CD $[17,18]$. Therefore, in the absence of specific biomarkers for these enteropathies, a duodenal biopsy still remains the gold standard for their diagnosis and for a prognostic evaluation by performing molecular testing, such as clonality for gamma T-cell receptor or flow cytometry analysis of intraepithelial lymphocytes [18].

\section{Annalisa Schiepatti ${ }^{1,2}$, David S Sanders ${ }^{1}$}

1) Academic Department of Gastroenterology, Royal Hallamshire Hospital, Sheffield, UK; 2) Gastroenterology Unit of IRCCS Pavia Institute, Istituti Clinici Scientifici Maugeri, University of Pavia, Italy

Corresponding to: David S Sanders, david.sanders1@nhs.net

Conflicts of interest: None.

DOI: http://dx.doi.org/10.15403/jgld-2548

\section{REFERENCES}

1. Shanahan W, Shanahan J. Inaction on IgA anti-tissue transglutaminase positivity. J Gastrointestin Liver Dis 2020;29 29(2):273-274. doi:10.15403/jgld-2297

2. Hopper AD, Hadjivassiliou M, Hurlstone DP, et al. What is the role of serologic testing in celiac disease? A prospective, biopsy-confirmed study with economic analysis. Clin Gastroenterol Hepatol 2008;6:314320. doi:10.1016/j.cgh.2007.12.008

3. Lebwohl B, Tennyson CA, Holub JL, Lieberman DA, Neugut AI, Green PH Sex and racial disparities in duodenal biopsy to evaluate for celiac disease. Gastrointest Endosc 2012;76:779-785. doi:10.1016/j.gie.2012.05.011

4. Sanders DS, Hurlstone DP, Stokes RO, et al. Changing face of adult coeliac disease: experience of a single university hospital in South Yorkshire. Postgrad Med J 2002;78:31-33. doi:10.1136/pmj.78.915.31

5. Husby S, Koletzko S, Korponay-Szabó I, et al. European Society Paediatric Gastroenterology, Hepatology and Nutrition Guidelines for Diagnosing Coeliac Disease 2020. J Pediatr Gastroenterol Nutr 2020;70:141-156. doi:10.1097/MPG.0000000000002497

6. Ludvigsson JF, Bai JC, Biagi F, et al; BSG Coeliac Disease Guidelines Development Group; British Society of Gastroenterology. Diagnosis and management of adult coeliac disease: guidelines from the British Society of Gastroenterology. Gut 2014;63:1210-1228. doi:10.1136/ gutjnl-2013-306578

7. Lock RJ, Stevens S, Pitcher MCL, Unsworth DJ. Is immunoglobulin A anti-tissue transglutaminase antibody a reliable serological marker of coeliac disease? Eur J Gastroenterol Hepatol2004;16:467-470. DOI: 10.1097/00042737-200405000-00005.

8. Clemente MG, Musu MP, Frau F, Lucia C, De Virgiliis S. Antitissue transglutaminase antibodies outside celiac disease. J Pediatr Gastroenterol Nutr2002;34:31-4

9 .Vecchi MD, Folli C, Donato MF, Formenti S, Arosio E, de Franchis R. High rate of positive anti-tissue transglutaminase antibodies in chronic liver disease. Role of liver decompensation and of the antigen source. Scand J Gastroenterol 2003;38:50-54. doi:10.1080/00365520310000438

10. Peracchi $M$, Trovato $C$, Longhi $M$, et al. Tissue transglutaminase antibodies in patients with end-stage heart failure. Am J Gastroentero 2002;97:2850-2854
11. Ferrara F, Quaglia S, Caputo I, et al. Anti-transglutaminase antibodies in non-coeliac children suffering from infectious diseases. Clin Exp Immunol 2010;159:217-223. doi:10.1111/j.1365-2249.2009.04054.x

12. Abrams JA, Diamond B, Rotterdam H, Green PH. Seronegative celiac disease: increased prevalence with lesser degrees of villous atrophy. Dig Dis Sci 2004;49:546-550. doi:10.1023/b:ddas.0000026296.02308.00

13. Tursi A, Brandimarte G, Giorgetti GM. Prevalence of antitissue transglutaminase antibodies in different degrees of intestinal damage in celiac disease. J Clin Gastroenterol 2003;36:219-221. doi:10.1097/00004836-200303000-00007

14. Hopper AD, Cross SS, Hurlstone DP, et al. Pre-endoscopy serological testing for coeliac disease: evaluation of a clinical decision tool. BMJ 2007;334:729. doi:10.1136/bmj.39133.668681.BE

15. Mooney PD, Wong SH, Johnston AJ, Kurien M, Avgerinos A, Sanders DS. Increased Detection of Celiac Disease With Measurement of Deamidated Gliadin Peptide Antibody Before Endoscopy. Clin Gastroenterol Hepatol 2015;13:1278-1284.e1. doi:10.1016/j. cgh.2015.01.010

16. Schiepatti A, Sanders DS, Biagi F. Seronegative coeliac disease: clearing the diagnostic dilemma. Curr Opin Gastroenterol 2018;34:154-158. doi:10.1097/MOG.0000000000000436

17. Schiepatti A, Biagi F, Fraternale G, et al. Short article: Mortality and differential diagnoses of villous atrophy without coeliac antibodies. Eur J Gastroenterol Hepatol 2017;29:572-576. doi:10.1097/ MEG.0000000000000836

18. Schiepatti A, Sanders DS, Aziz I, et al. Clinical phenotype and mortality in patients with idiopathic small bowel villous atrophy: a dual-centre international study. Eur J Gastroenterol Hepatol 2020 Apr 10. doi:10.1097/MEG.0000000000001726

\section{Challenges of pancreatic cancer chemotherapy in the COVID-19 era}

\section{To the Editor,}

The recent pandemic of coronavirus disease 2019 (COVID-19) has challenged the oncology medical community, regarding the treatment for these patients. China reported that the rate of severe acute respiratory syndrome coronavirus 2 (SARS-CoV-2) infection was higher in oncological patients (1\%) compared to the general population (0.29\%), with higher rates of respiratory complications [1]. The risk factors for severe respiratory complications were: recent past medical history of chemotherapy or surgery $(\mathrm{OR}=5.34, \mathrm{p}=0.0026)$.

Pancreatic cancer (PC) is a malignancy with a high mortality, being the fourth leading cause of cancer related deaths in the United States and the sixth leading cause of cancer related deaths in Romania [2]. In locally advanced and metastatic pancreatic cancer (LAMPC) chemotherapy might determine conversion to resectable stages and prolong the overall survival (OS) [3]. Recent guidelines recommend oncologists to maintain optimal but safe cancer care and avoid the distraction effect [4].

Our experience consisted of eleven patients with LAMPC, enrolled at the Regional Institute of Gastroenterology and Hepatology, Cluj-Napoca, Romania, at the time of the COVID 19 outbreak in Romania. The demographic data are summarized in Table I. The patients were with good performance status 
$(\mathrm{ECOG}=0-1)$, with a median age of 62 years. Nine patients had at least one comorbidity at diagnosis (high blood pressure, diabetes mellitus, chronic ischemic heart disease). At the onset of the COVID-19 pandemic, nine patients were on chemotherapy and two patients were on the initial work-up.

In order to minimize the exposure, we switched the chemotherapy regiment from FOLFIRINOX protocol (oxaliplatin, calcium leucovorin, irinotecan, 5-fluorouracil (5-FU)) to gemcitabine-based chemotherapy: five patients were switched from FOLFIRINOX to GEMCAP regimen (gemcitabine, capecitabine) and one patient to Gemcitabine monotherapy. Two patients that were on GEMOX regimen (gemcitabine, oxaliplatin) were switched to gemcitabine monotherapy. The patient with leucovorin and 5-FU maintenance therapy was switched to capecitabine maintenance monotherapy. The two chemonaive patients started directly with the GEMCAP regimen. All patients tolerated the treatment well. The number of hospital admission and continuous hospital days decreased. During the new chemotherapy regimen none of the patients was infected with COVID-19.

In the COVID-19 era, the assessment risk/benefits of chemotherapy in LAMPC patients is stressed by the risk factors for severe infection. Chemotherapy might determine a weaker immune system. Other risk factors for severe infections are older age and the presence of comorbidities as in our cohort.

The European Society for Medical Oncology (ESMO) recommends initiation and continuation of first line chemotherapy in patients fit for a combined regimen, the goal being the improvement of quality of life and OS [5]. For patients with good performance status the standard chemotherapy is represented by combined regimens based on gemcitabine or 5-FU and for patients with poor performance status by gemcitabine alone $[6,7]$.

Gemcitabine alone has been the standard treatment in LAMPC since 1997, considering the overall survival benefit and the clinical benefit compared to 5-FU [3]. Several trials investigated the association of gemcitabine with other chemotherapeutical agents (irinotecan, 5-FU, cisplatin, oxaliplatin or capecitabine) and reported contradictory results. The association of gemcitabine with capecitabine or cisplatin showed the biggest benefits [4]. Gemcitabine and capecitabine showed a trend towards the improvement of OS when compared to gemcitabine [8]. A recent meta-analysis that included 8 trials with gemcitabine and capecitabine versus gemcitabine monotherapy showed improved OS for combined regimen $(\mathrm{HR}=0.87, \mathrm{p}=0.03)$ [9]. The preferred chemotherapy regimen for first line treatment is gemcitabine and nab-paclitaxel based on the results of the phase III trial MPACT in which OS was significantly improved with this association compared to gemcitabine alone (OS 8.7 months vs 6.6 months, $H R=0.72, p<0.001$ ) [10]. In patients with good performance status, the recommended chemotherapy for first line treatment is represented by the triplet FOLFIRINOX. The PRODIGE trial randomized between FOLFIRINOX and gemcitabine monotherapy and reported better OS overall in the FOLFIRINOX arm (11 months vs 6.8 months, $\mathrm{p}<0.001$ ), but with more toxicities [11].

Based on guidelines, and on our experience regarding the benefits and risk of chemotherapy treatment in LAMPC patients in COVID-19 pandemic we propose the switch from FOLFIRINOX regimen to gemcitabine-based regimens or to initiate gemcitabine based first line chemotherapy.

Radu Vidra ${ }^{1,2}$, Raluca Bodea ${ }^{2}$, Voicu Mercea ${ }^{1,2}$, Adina Nemes ${ }^{1,3}$

1) Iuliu Hatieganu University of Medicine and Pharmacy Cluj-Napoca; 2) Regional Institute of Gastroenterology and Hepatology ClujNapoca; 3) Oncology Institute Cluj-Napoca, Romania.

Corresponding to: Radu Vidra, raduvidra@gmail.com

Conflicts of interest: None.

DOI: http://dx.doi.org/10.15403/jgld-2527

Table I

\begin{tabular}{|c|c|c|c|c|c|c|c|c|c|c|}
\hline Patient & Gender & $\begin{array}{c}\text { Age at } \\
\text { diagnostic }\end{array}$ & $\begin{array}{l}\text { Histology } \\
\text { grade }\end{array}$ & TNM & $\begin{array}{c}\text { Sites of metastatic } \\
\text { disease }\end{array}$ & Stage & $\begin{array}{c}\text { Prior } \\
\text { Therapies }\end{array}$ & $\begin{array}{l}\text { No. of } \\
\text { cycles }\end{array}$ & $\begin{array}{l}\text { ECOG } \\
\text { status }\end{array}$ & New Therapy \\
\hline 1 & male & 53 & 2 & cT3N1M1 & liver, peritoneum & IV & FOLFIRINOX & 2 & 1 & GEMCAP \\
\hline 2 & male & 62 & 3 & cT4N2M1 & liver & IV & LV5FU2* & 4 & 1 & Capecitabine \\
\hline 3 & male & 64 & 3 & cT4N1M1 & peritoneum & IV & FOLFIRINOX & 2 & 1 & GEMCAP \\
\hline 4 & male & 65 & 3 & cT3N1M1 & liver, peritoneum & IV & FOLFIRINOX & 1 & 1 & GEMCAP \\
\hline 5 & female & 54 & 3 & cT4N1M0 & none & III & FOLFIRINOX & 3 & 1 & GEMCAP \\
\hline 6 & male & 71 & 2 & pT2N2cMo & $\begin{array}{l}\text { lymph nodes } \\
\text { relapse }\end{array}$ & III & FOLFIRINOX & 6 & 1 & GEMCAP \\
\hline 7 & male & 58 & 3 & pT2N1cM0 & $\begin{array}{l}\text { lymph nodes } \\
\text { relapse }\end{array}$ & IIB & FOLFIRINOX & 12 & 1 & Gem \\
\hline 8 & male & 61 & 3 & cT4N1M1 & liver & IV & none $e^{\star *}$ & $\mathrm{n} / \mathrm{a}$ & 1 & GEMCAP \\
\hline 9 & female & 67 & 3 & cT4N1M1 & lung & IV & none $e^{\star *}$ & $\mathrm{n} / \mathrm{a}$ & 1 & GEMCAP \\
\hline 10 & male & 56 & 3 & cT4N1M0 & none & III & GEMOX & 8 & 1 & Gem \\
\hline 11 & female & 66 & 3 & cT3N1M0 & none & III & GEMOX & 15 & 1 & Gem \\
\hline
\end{tabular}

FOLFIRINOX protocol: oxaliplatin, calcium leucovorin, irinotecan, 5-fluorouracil (5-FU); Gem: gemcitabine; GEMCAP regimen: gemcitabine, capecitabine; GEMOX regimen: gemcitabine, oxaliplatin; LV5FU2: leucovorin and 5-FU; n/a=not assessed. ${ }^{*}$ LV5F2 maintenance therapy after 12 cicles of FOLFIRINOX ${ }^{* *}$ chemonaive new patients 


\section{REFERENCES}

1. Liang W, Guan W, Chen R, et al. Cancer patients in SARS-CoV-2 infection: a nationwide analysis in China. Lancet Oncol 2020;21:335337. doi:10.1016/S1470-2045(20)30096-6

2. World Health Organization. International Agency for Research on Cancer. Globocan 2018. Romania. Available at:https://gco.iarc.fr/today/ data/factsheets/populations/642-romania-fact-sheets.pdf

3. Burris HA 3rd, Moore MJ, Andersen J, et al. Improvements in survival and clinical benefit with gemcitabine as first- line therapy for patients with advanced pancreas cancer: A randomized trial. J Clin Oncol 1997;15:2403-2413. doi:10.1200/JCO.1997.15.6.2403

4. Ducreux M, Cuhna AS, Caramella C, et al. Cancer of the pancreas: ESMO Clinical Practice Guidelines for diagnosis, treatment and followup. Ann Oncol 2015;26 Suppl 5:v56-v68. doi:10.1093/annonc/mdv295

5. ESMO. Cancer Patient Management During the COVID-19 Pandemic. ESMO management and treatment adapted recommendations in the covid-19 era: pancreatic cancer. Available at: https://www.esmo. org/guidelines/cancer-patient-management-during-the-covid-19pandemic/gastrointestinal-cancers-pancreatic-cancer-in-the-covid19-era. Accessed 03 May 2020.

6. National Comprehensive Cancer Network. NCCN Guidelines 1395. Available at: https://www.nccn.org/professionals/physician_gls/pdf/ pancreatic.pdf. Accessed: 02 May 2020.

7. ESMO. Pancreatic Cancer. Cancer of the pancreas: esmo clinical practice guidelines. Available at: https://www.esmo.org/guidelines/ gastrointestinal-cancers/pancreatic-cancer. Accessed: 13 Apr 2020.

8. Cunningham D, Chau I, Stocken DD, et al. Phase III randomized comparison of gemcitabine versus gemcitabine plus capecitabine in patients with advanced pancreatic cancer. J Clin Oncol 2009;27:55135518. doi:10.1200/JCO.2009.24.2446

9. Li Q, Yan H, Liu W, Zhen H, Yang Y, Cao B. Efficacy and safety of gemcitabine-fluorouracil combination therapy in the management of advanced pancreatic cancer: A meta-analysis of randomized controlled trials. PLoS One 2014;9:e104346. doi:10.1371/journal. pone. 0104346

10. Von Hoff DD, Ervin T, Arena FP, et al. Increased survival in pancreatic cancer with nab-paclitaxel plus gemcitabine. N Engl J Med 2013;369:1691-1703. doi:10.1056/NEJMoa1304369

11. Conroy T, Desseigne F, Ychou M, et al. "FOLFIRINOX versus gemcitabine for metastatic pancreatic cancer. N Engl J Med 2011;364:1817-1825. doi:10.1056/NEJMoa1011923

\section{Pancreatic involvement in SARS-CoV-2: case report and living review}

\section{To the Editor,}

Since the novel coronavirus outbreak started, as of May, 22, 2020, 5,067,579 individuals have been confirmed with COVID-19 and 332,711 of them have died from the disease [1]. Besides respiratory involvement, a meta-analysis of 60 studies reporting on 42,443 coronavirus disease 2019 patients proved that $18 \%$ of them had gastrointestinal symptoms [2]. Recently, it has been suggested that severe acute respiratory syndrome coronavirus 2 (SARS-CoV-2) infection, similar to other viral infections, might induce pancreatic injury or even worse, acute pancreatitis.
We report the case of a 47-year-old male patient with COVID-19, without significant medical history or home medication, who only complained of a dry cough for a week. Initial physical examination and chest x-ray did not show any abnormal changes. Admission laboratory work-up revealed mild lymphopenia $(1,420 / \mathrm{mm} 3)$, increased inflammatory marker levels [C reactive protein (CRP) $31 \mathrm{mg} / \mathrm{l}$, erythrocytes sedimentation rate (ESR) $64 \mathrm{~mm} / \mathrm{h}$, ferritin $583 \mathrm{ng} / \mathrm{ml}$ ], procalcitonin and $\mathrm{D}$-dimers within normal range, minimal elevation in gama glutamil transpeptidase (GGT) $(2.4 \times \mathrm{ULN})$ and alanine aminotransferase (ALT) $(1.3 \times \mathrm{ULN})$ in the absence of viral hepatitis B or C.

During the following days regular blood tests came back normal and the cough faded. On day 12 he complained of severe epigastric pain with radiation to the back, nausea, constipation and lack of flatus. Abdominal examination revealed epigastric tenderness and bloating, while blood tests showed mild leukocytosis with lymphopenia, increased pancreatic enzymes (lipase $22 \mathrm{x}$ upper normal limits (ULN), amylase $6 \mathrm{x}$ ULN) and CRP (63.24 mg/l). Procalcitonin along with liver and kidney function tests were within the normal range. Chest and abdomen computed tomography scans revealed the presence of scattered bilateral subpleural groundglass opacities, edematous changes in the caudal region of the pancreas (Fig. 1a) and bowel distention without evidence of obstruction (Fig. 1b). According to the modified Atlanta criteria the patient was diagnosed with acute pancreatitis complicated by ileus.

Other causes of acute pancreatitis, such as gallstones/ biliary obstruction, alcoholism, smoking, drugs, trauma, hypertriglyceridemia and hypercalcemia were excluded. Since no other plausible cause was found for both acute pancreatitis and ileus, and sustained by the evolution, we concluded that SARS-CoV-2 infection might be responsible. He was managed conservatively with bowel rest, intravenous crystalloid fluid resuscitation, prokinetic medication and analgesia. After 2 days colonic transit normalized, and pancreatic enzymes gradually decreased.

There is evidence that the virus remains in the gastrointestinal tract, perhaps in the pancreas as well, even after negative nasopharyngeal swabs had become negative [2, 3], possibly explaining the delayed onset of pancreatitis in our patient.

Since there is hardly any data on pancreatic involvement in SARS-CoV-2 infection, an extensive literature search was performed on 21 May 2020 in six major databases: PubMed, Web of Science, Springer, Ovid, Scopus, Wiley, using the terms: "pancreatitis", "pancreatic injury", "lipase", "amylase", "COVID-19" and "SARS-CoV-2". The initial search yielded 160 articles. After title and abstract screening, removing duplicates and assessment for eligibility, we identified a total of 4 articles [4-7], comprising thirteen COVID-19 patients, labeled either as pancreatic injury (9/13) or acute pancreatitis (4/13). Since some of the reported cases lacked in details regarding diagnostic criteria, only two $(2 / 13)$ of the cases might be interpreted as acute pancreatitis based on the revised Atlanta classification. In a retrospective study, 13/121 patients with COVID-19 had increased pancreatic enzymes, but none had values above 3 $\mathrm{x}$ ULN [3]. 


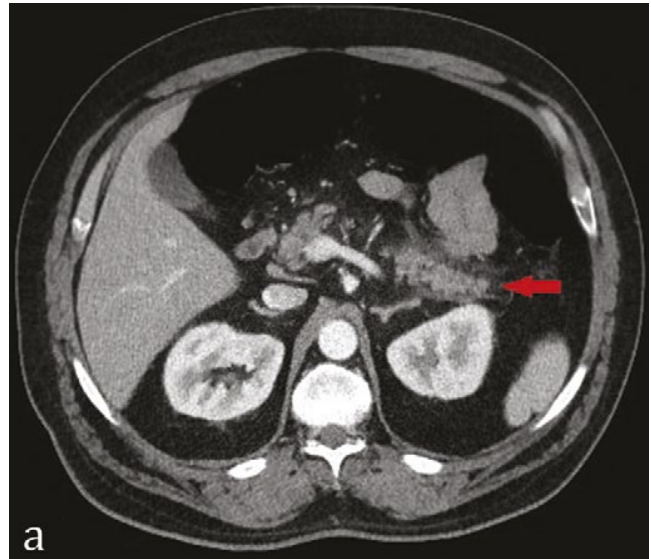

Fig. 1a. Blurring of the pancreatic contours due to the edema of the surrounding adipose tissue.

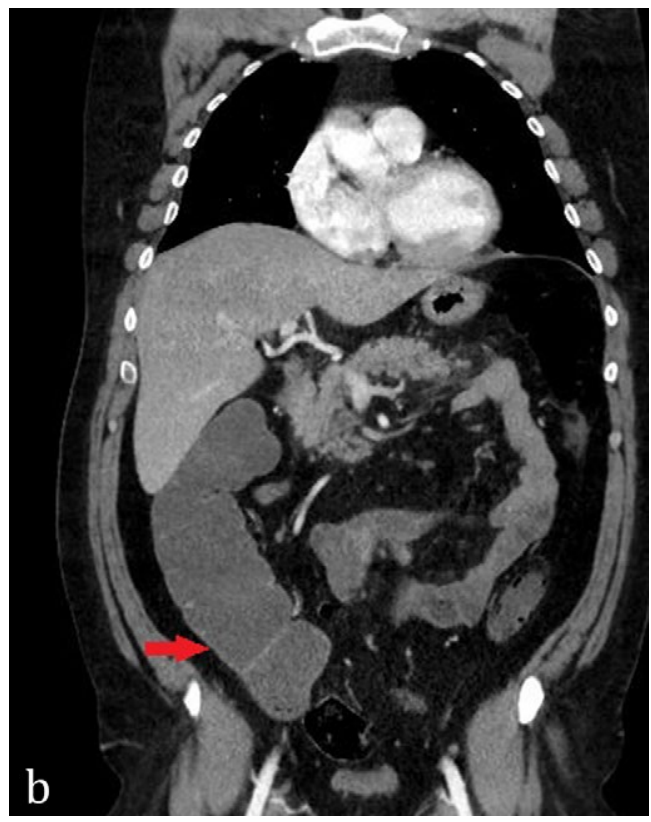

Fig. 1b. Cecum and ascending colon distension due to ileus.

Whereas some authors support the theory that increased pancreatic enzymes in COVID-19 patients might not suggest pancreatic injury at all, and due to the clinical context additional causes should be considered, we suggest that some patients might indeed have at least a mild pancreatic injury.
However, since there is still limited evidence of pancreatic involvement in SARS-CoV-2 infection, no definite conclusions can be made. We mitigate that the potential for pancreatic injury still remains, and all patients with features suggestive of acute pancreatitis should be properly investigated. Nevertheless, abnormal pancreatic enzymes should only be interpreted as acute pancreatitis based on the revised Atlanta classification.

\section{Larisa Pinte ${ }^{1}$, Cristian Băicuș ${ }^{1,2}$}

1) Internal Medicine Department, Colentina Clinical Hospital, Bucharest; 2) Carol Davila University of Medicine and Pharmacy, Bucharest, Romania

\section{Corresponding to: Larisa Pinte, larissa.0806@yahoo.com}

Conflicts of interest: None.

DOI: http://dx.doi.org/10.15403/jgld-2618

\section{REFERENCES}

1. European Centre for Disease Prevention and Control (ECDC). Download today's data on the geographic distribution of COVID-19 cases worldwide. Available at: https://www.ecdc.europa.eu/en/ publications-data/download-todays-data-geographic-distributioncovid-19-cases-worldwide. Accessed 2020 May 22.

2. KAHI CJ. Gastrointestinal Aspects of COVID-19. NEJM Journal Watch Gastroenterology 2020 Apr 3. Available at: https://www.jwatch.org/ na51324/2020/04/09/gastrointestinal-aspects-covid-19

3. Xiao F, Tang M, Zheng X, Liu Y, Li X, Shan H. Evidence for Gastrointestinal Infection of SARS-CoV-2. Gastroenterology 2020;158:1831-1833.e3. doi:10.1053/j.gastro.2020.02.055

4. Anand ER, Major C, Pickering O, Nelson M. Acute pancreatitis in a COVID-19 patient. Br J Surg 2020;107:e182. doi:10.1002/bjs.11657

5. Aloysius MM, Thatti A, Gupta A, Sharma N, Bansal P, Goyal H. COVID-19 presenting as acute pancreatitis. Pancreatology 2020 May 8. doi:10.1016/j.pan.2020.05.003

6. Hadi A, Werge M, Kristiansen KT, et al. Coronavirus Disease-19 (COVID-19) associated with severe acute pancreatitis: Case report on three family members. Pancreatology 2020 May 5. doi:10.1016/j. pan.2020.04.021

7. Wang F, Wang H, Fan J, Zhang Y, Wang H, Zhao Q. Pancreatic injury patterns in patients with COVID-19 pneumonia. Gastroenterology 2020 Apr 1. doi:10.1053/j.gastro.2020.03.055 